\title{
Kalman Filter Effective to Hydrologic Routing?
}

\author{
Yang-Liang Jeng \\ Associate Professor, Department of Mechanical and Marine Engineering National Taiwan Ocean University Keelung, \\ Taiwan R.O.C.
}

Rong-Che Chen

Associate Professor, Department of Mechanical and Marine Engineering National Taiwan Ocean University Keelung, Taiwan R.O.C.

Chao-He Chang

Associate Professor, Department of Mechanical and Marine Engineering National Taiwan Ocean University Keelung, Taiwan R.O.C.

Follow this and additional works at: https://jmstt.ntou.edu.tw/journal

Part of the Mechanical Engineering Commons

\section{Recommended Citation}

Jeng, Yang-Liang; Chen, Rong-Che; and Chang, Chao-He (2009) "Kalman Filter Effective to Hydrologic Routing?," Journal of Marine Science and Technology. Vol. 7: Iss. 1, Article 7.

DOI: $10.51400 / 2709-6998.2512$

Available at: https://jmstt.ntou.edu.tw/journal/vol7/iss1/7

This Research Article is brought to you for free and open access by Journal of Marine Science and Technology. It has been accepted for inclusion in Journal of Marine Science and Technology by an authorized editor of Journal of Marine Science and Technology. 


\section{Kalman Filter Effective to Hydrologic Routing?}

\section{Acknowledgements}

This work was supported by the National Science Council, R.O.C. under Grant \#86-2212-E-019-005 and \#87-2212-E-019-008 


\title{
STUDIES OF TUMBLING MOTION GENERATED DURING INTAKE IN A BOWL-IN-PISTON ENGINE
}

\author{
Yang-Liang Jeng*, Rong-Che Chen* and Chao-He Chang
}

Keywords: Engine, Tumbling Motion, Particle Image Velocimetry, Flow Structure.

\section{ABSTRACT}

Intake-generated tumbling motion has been recently proposed to enhance the turbulence level during compression stroke to promote the combustion rate and reduce operation variations. This study is to investigate the quality of the tumbling motion, especially for the engine with a bowl-in-piston. To overcome the difficulties on the flow visualization caused by the high-speed motion of a real engine, a transparent water-analogy-simulation system is designed based on similarity analysis and fabricated to model the operation of an actual engine. Benchmark tests including the qualitative flow visualization and quantitative full-field measurements using a high-speed PIA system have been conducted to study the time-varying nature of the tumbling motion within the cylinder. The results demonstrate the success on experimental set-ups and the potential of using the current system in studying the complex engine intake-generated tumbling motion.

\section{INTRODUCTION}

In recent years, fossil fuel has been extensively used on prime movers. Accompanied by the economical progress and civilization, the level of air pollution of combustion products has been raised. The environmental pollution becomes serious enough to be a muchconcerned issue. The emissions of exhaust gases are also responsible for the green house effects of the atmosphere. These effects lead to changes in the global climate, which may result in a crisis of abrupt change in the biological environment.

Among all the efforts being made to reduce the emissions of internal combustion engines, the lean-burn strategy has been proved effective. However, the burning speed of a lean fuel-air mixture is slower, which reduces the engine performance and constrains the im-

Paper Received May 5, 1999. Accepted June 22, 1999. Author for Correspondence: Yang-Liang Jeng.

*Associate Professor, Department of Mechanical and Marine Engineering National Taiwan Ocean University Keelung, Taiwan R.O.C. provement in engine emissions. Therefore, spark ignition engines adopting the lean-burn strategy usually require a higher compression ratio and a more compact combustion chamber to promote the speed of flame propagation (Heywood, 1988). Adopting other fuel such as CNG is an alternative to improve engine emissions (Evans and Blaszczyk, 1997). Cummins engine has combined both the two above-mentioned approaches (Duggal, 1996). Diesel engines with a bowl-in-piston have been modified to fuel with CNG. Air-fuel mixture is burned at a lean ratio to improve engine emissions.

The drawbacks of lean-burn engines in the slow combustion speed and the low engine performance may be improved under adequate in-cylinder flow motions. When the turbulence intensity of the in-cylinder flow increases, the flammable range of the premixed combustible gases is broadened. Accordingly, the fuel-air mixture becomes easier to be ignited such that the flame propagation speed is increased and the cyclic variability is significantly reduced. Shortening in the combustion duration will make the engine to possess the potential to be operated at higher speeds and to develop higher engine outputs.

Generation of swirling motion, which is a rotational motion of inducted charge about the axis parallel to cylinder axis, has been traditionally considered in the engine combustion chamber designs. Intake-generated swirling flow may be created by special designs of intake port or by covering up part of the peripheral valve opening area. Helical ports are often constructed when the swirling flow of intake charge is to be generated through the configurations of ports. Other approaches proposed are the uses of directed intake port, shrouded inlet valve or masked cylinder head. For the above proposals, inducting flow is restricted in a desired direction through the intake valve opening, such that tangential flows to result in a swirling motion is generated within the cylinder. During the earlier half of intake process, the swirling flow within the cylinder does not distribute uniformly. The angular velocity increases rapidly with the intake valve lift and the piston speed. The increase of swirl velocity then slows 
down in the later half of intake stroke. The flow pattern gradually turns close to a solid-body rotation with the tangential velocity increasing with the increase in radius (Heywood, 1987; 1988). As the piston motion slows down, the flow pattern redistributed, with the speed of swirl close to piston decreasing. Thereafter, the angular momentum of swirl motion gradually decreases due to the turbulence dissipation caused by viscous effect. Swirl ratio is defined as the angular velocity of solid-body rotating flow divided by the angular velocity of rotating crankshaft. Swirl ratio is proportional to the angular momentum, but inversely proportional to the moment of inertia. During compression stroke, swirl ratio decreases with the decay of angular momentum. When the piston moves close to the top dead center (TDC), the variation of swirl ratio depends on the shape of combustion chamber. For disc or wedge-typed combustion chambers with flattop piston, swirl ratio decreases continually during compression. For combustion chambers with bowl-in-piston, the gases are squished into piston bowl when the piston moves close to the TDC. The moment of inertia decreases abruptly, leading to the increase of swirl ratio (Belair et al., 1983). This increase in the large-scale flow speed contributes to the fuel spray being spread out in diesel engines, which accelerates the processes of the fuel-air mixing and therefore the combustion.

Another intake-generated large-scale vortical flow pattern is the tumbling motion. The rotation axis of vortex is normal to the cylinder axis, resembling the rotation of a barrel. Thus, tumbling motion is also called as vertical swirl or barrel swirl. Similar to swirling flow, tumbling flow may be generated by special designs of the intake port. Utilization of a shrouded inlet valve or a masked cylinder head may also be proposed to generate in-cylinder tumbling flow. To generate a pure tumbling motion for single intake-valve cylinders, the directional vector of intake jet should be on the plane defined by the cylinder axis and the intake valve axis. A tumbling flow with both the radial and the axial motion is therefore expected in the cylindrical coordinate. If an intake jet has only the tangential and the axial components in the cylindrical coordinate, a pure swirl flow is produced. Tipped swirl (or called inclined swirl/tumble) with a characteristic angle between the rotating axis and the cylinder axis is generated for the intake jet with all three components, (e.g., Kent et al, 1989; Arcoumanis, 1990; Khalighi and Huebler, 1988).

Researches have been done on the tumbling motions in spark ignition engines. Kent et al. (1989) have conducted flow visualization on the in-cylinder flow fields during intake stroke. It is found that the threedimensional flow field, with the rotation axis of the tipped swirl inclined from cylinder axis by $30^{\circ}$, at the bottom dead center (BDC) contributes to an increase in the turbulence level during the compression process. Conclusion was drawn that the enhancement in the vortex generated by tumbling motion will shorten the duration of combustion process (Kent et al, 1989). Through LDA measurements, Arcoumanis et al. (1990) found that a pure tumbling motion is able to produce a $42 \%$ of enhancement in turbulence intensity, while a $24 \%$ of turbulence enhancement can be achieved under a combined tumbling/swirling vortical flow particularly generated in their experiments. The higher the swirl velocity, the more the turbulent kinetic energy is dissipated from the decay of the large-scale motion during compression. Khalighi and Huebler (1988) found that the tumbling vortex, attained through the sole use of an intake jet, has a radius less than half of the cylinder bore. With the application of a shrouded intake valve, a strong tumbling vortex may be generated to fill the whole cylinder steadily, which does not decay easily by the turbulence dissipation. They suggested that an analog definition of the tumble ratio as the ratio of vortex angular velocity to crankshaft angular velocity. Khalighi $(1990$; 1991; 1995) further conducted the velocity measurements using PIV in an analog model engine to calculate the tumbling ratio. It is found that, for spark ignition engines, the tumble ratios at the BDC are around 2.8 without changing with the engine speed.

Though there starts to see researches on in-cylinder tumbling motion for spark ignition engines with flat-topped piston, there has no open literature concerning the tumbling motion for engines with bowl-inpiston. It calls for the needs to investigate the flow structure of the intake-generated tumbling motion and the relationship with the turbulence produced after compression. This article presents the preliminary results in a series of research on the in-cylinder flow field. During the preliminary study, an analog simulation is performed to construct a transparent analog model engine. Laser flow visualization on the model engine in conducted to study qualitatively the variations of the tumble flow structure under different crank angles during the intake process. Khalighi and Huebler (1988) and Kent et al. (1989) have demonstrated the potential of using PIV in the studies of the in-cylinder flow fields. Therefore, the high-speed particle image analyzer developed by Chen and Chou (1998) is also adopted in this research for measuring the quantitative information.

\section{MODEL ENGINE SYSTEM}

Being highly time-variant, the in-cylinder flow field would be difficult to be characterized by using a time-averaged point-measurement technique. A tech- 
nique with the ability of field measurement is necessary for the study of in-cylinder flow structure. Techniques based on video recording should be the most convenient methods to accomplish the task of field measurement. However, the flow inside an actual engine is too fast to be captured by a traditional video system. It is therefore necessary to build, based on the similarity analysis, a similar engine model operating at a much lower speed. This similar engine model bears similar average flow characteristics to that in an actual engine. In the meantime, a measurement method with high imagerecording rate should be adopted. The instrumentation of measurement will be discussed in the next section. The set-up of model engine system is described below in details. However, one must keeps in mind that only the average flow field can be simulated by the similarity law. The detailed turbulence properties, which dominate the mixing and combustion efficiency, must be studied by other methods.

\section{Similarity Analysis}

Adopting an optical technique as the measurement method, a transparent engine model is necessary for this study. To build a similar engine model based on the similarity analysis, the first step is to replace the working fluid. Due to its availability, stability and the nontoxic characters, water is considered as the best fluid. In order that a water analogy system may fully simulate an actual engine system, at least four similarity criteria must be satisfied (Willas et al., 1966). The geometric similarity is automatically complied since the physical dimensions of model engine are chosen the same as those of an actual engine. It should be noted that no compression or combustion strokes are involved in the current work. Therefore, no thermal similarity should be considered. The remaining two similarity relations are discussed below.

\section{Dynamic similarity}

Considering the flow as incompressible (will be proved later) during the intake stroke, two important parameters emerge as one looks into the dimensionless form of the Navier-Stoke's equation. These are Reynolds number and Strouhal number

$$
\begin{aligned}
& \operatorname{Re}=\frac{U_{p} D}{v_{f}} \approx \frac{\text { inertia force }}{\text { viscous force }}, \\
& S t=\frac{D}{t U_{p}} \approx \frac{\text { inertia force in the vortical region }}{\text { inertia(convective) force }},
\end{aligned}
$$

where $U_{p}$ is the averaged piston speed, $D$ is the bore size, $v_{f}$ is the kinematic viscosity of liquid and $t$ represents a proper time scale. The dynamic similarity builds up when the Reynolds and Strouhal numbers are matched for both the model and the actual engines, that is

$$
\begin{aligned}
& \left(\frac{U_{p} D}{v_{f}}\right)_{\text {engine }}=\left(\frac{U_{p} D}{v_{f}}\right)_{\text {model }}, \\
& \left(\frac{D}{t U_{p}}\right)_{\text {engine }}=\left(\frac{D}{t U_{p}}\right)_{\text {model }} .
\end{aligned}
$$

Since the sizes of the model and the actual engines are the same and water is adopted as the working fluid, Eqs. (3) and (4) become

$$
\begin{aligned}
& \left(\frac{U_{p}}{v_{\text {air }}}\right)_{\text {engine }}=\left(\frac{U_{p}}{v_{\text {water }}}\right)_{\text {model }}, \\
& \left(t U_{p}\right)_{\text {engine }}=\left(t U_{p}\right)_{\text {model }} .
\end{aligned}
$$

The proper time scale $t$ may be chosen as the cyclic period of the crankshaft motion, which is inversely proportional to the angular velocity of crankshaft $\omega_{c}$. The similarity in Strouhal number can then be derived as

$$
\left(\frac{U_{p}}{\omega_{c}}\right)_{\text {engine }}=\left(\frac{U_{p}}{\omega_{c}}\right)_{\text {model }},
$$

Since the averaged piston speed $U_{p}$ is proportional to $\omega_{c}$, Eq. (7) is automatically satisfied. The viscosity ratio between the water and the air is around 20. According to Eq. (5), this suggests that the operating speed of model engine can be $1 / 20$ of that of the actual engine. The fluid velocity of the in-cylinder flow field is therefore significantly reduced to render the possibility of image capture.

\section{Kinematic similarity}

The compressibility properties of the test fluid (water) and the air are different and there seems no way to match the kinematic similarity between each other perfectly. Estimation of the errors caused by the divergence in kinematic properties is necessary. It is well known that the change in density can be approximately examined as

$$
\frac{\Delta \rho_{f}}{\rho_{f}} \approx M^{2},
$$

where $\rho_{f}$ is the density of fluid and $M$ represents the Mach number. Since Mach number of the air-flow in an actual engine during the intake stroke is around 0.2 (Heywood, 1988), the ratio of density change is less than $5 \%$. The incompressibility of both fluids can be approximately assumed (Ma et al., 1986). In the mean time, the pressure change for the intake air can be estimated through the Euler number expressed as 


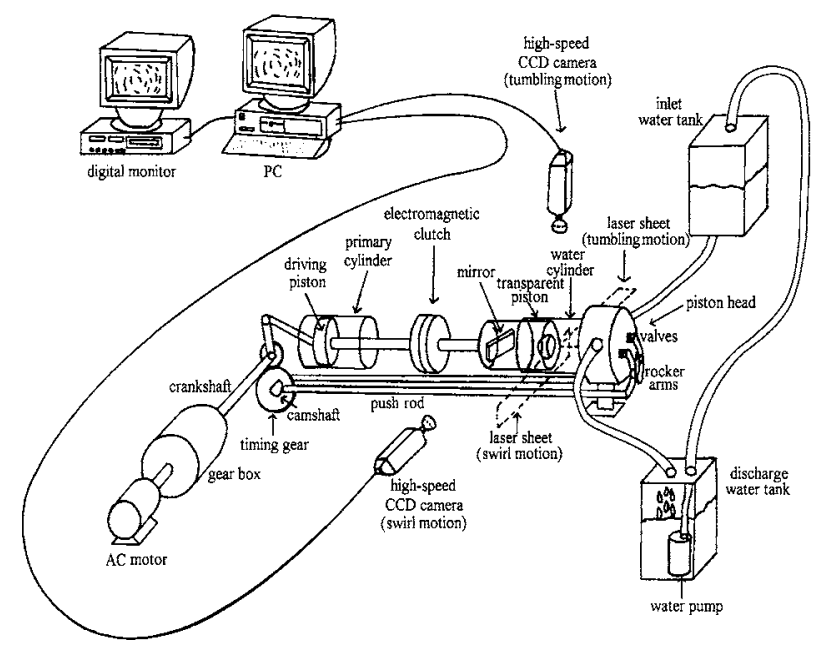

Fig. 1. Schematic diagram of the test facility.

(Zucrow and Haffman, 1976)

$$
E u=\frac{\Delta p}{\frac{1}{2} \rho_{f} V^{2}} \approx 1+\frac{M^{4}}{4}+\frac{M^{2}}{40}+\frac{M^{2}}{1600}+\ldots
$$

Euler number is close to unity when $M$ (or density change) is small. This results in the pressure difference for the entrained water in model engine being around twice of that for the air entrained in an actual engine. The variation in the flow structure due to this pressure difference between both incompressible fluids should be minor and can be neglected.

\section{Construction of Analogy Simulation System}

At the beginning stage of this research, a singlecylinder horizontal 4-stroke diesel engine has been chosen as the researching target. The main specifications of this engine are listed in Table 1. According to the similarity analysis in the last section, an analogy simulation system with transparent water cylinder is constructed to simulate the above-mentioned engine. The fundamental configuration of this simulation system is drawn schematically in Fig. 1. It includes, from left to right in Fig. 1, the following units or parts: driving unit, cranking mechanism, electromagnetic clutch, test section, cylinder head, and intake/exhaust unit. These units and parts are described below:

\section{Test unit}

The test unit includes a transparent water-cylinder and a transparent piston; both made of acrylic resin, a material without optical obstructions. Laser sheets are projected into the test section to illuminate the flow
Table 1. Specifications of the actual engine simulated

\begin{tabular}{lr}
\hline Brand/Model & YANMAR TF120F \\
Type & 4-stroke, horizontal \\
Number of cylinder & single cylinder \\
Bore/Stroke & $92 \mathrm{~mm} / 96 \mathrm{~mm}$ \\
Displacement volume & $0.638 \mathrm{~L}$ \\
Compression ratio & 17.1 \\
Rated speed & $2400 \mathrm{rpm}$ \\
Number of valves & 1 intake valve/1 exhaust valve \\
Intake valve head diameter/lift & $37 \mathrm{~mm} / 9.5 \mathrm{~mm}$ \\
Exhaust valve head diameter/lift & $31 \mathrm{~mm} / 9.5 \mathrm{~mm}$ \\
Intake valve timing & IVO@ $10^{\circ} \mathrm{BTC} / \mathrm{IVC} @ 33^{\circ} \mathrm{ABC}$ \\
Exhaust valve timing & EVO@30 BBC / EVC@ $5^{\circ} \mathrm{ATC}$ \\
\hline
\end{tabular}

field in the specified direction. Video cameras are then applied to track the seeding particles and resolve the incylinder flow fields from them. The transparent piston is connected to an electromagnetic clutch and a primary piston, which in fact is the original piston of the engine. Therefore, the transverse distance during intake stroke is kept the same as the original engine. The inner diameter of the transparent water-cylinder is $90 \mathrm{~mm}$, close to the bore of the original engine. To study the effects on tumbling motion influenced by different geometric aspect ratios of piston-bowl, the clearance volumes are purposely kept the same for different transparent pistons. Since laser sheets must pass across different media, image distortion and measuring inaccuracy will be encountered due to the difference in the refractive index. This image distortion difficulty will be especially serious for the light passing through a curved surface such as the cylinder wall. In order to avoid the measuring inaccuracy caused by image distortion, a refractive index matching technique must be applied. The transparent water-cylinder/piston assembly is enclosed in a rectangular box made of the transparent acrylic. Pure glycerin, of nearly the same refractive index as acrylic resin, is filled in between the transparent cylinder and the enclosed acrylic box.

The incidence direction of laser sheet depends on the interest of study of the flow motion. To observe the in-cylinder tumbling motion, laser sheet is projected horizontally into the transparent cylinder on the surface passing through the cylinder axis. A video camera is installed above the transparent cylinder, viewing at an angle normal to the incidence direction of the laser sheet. A high-speed particle image analyzer (PIA) system is used to analyze the recorded trails of tracking particles and to study the variations of the tumbling flow structure inside the transparent cylinder. 


\section{Driving unit}

The driving unit includes an $\mathrm{AC}$ motor and a transmission gearbox. The AC motor is 220 volt, 3 phase, 5HP, with a constant speed of $1750 \mathrm{rpm}$. The transmission gearbox reduces the rotating speed of motor and drives the cranking mechanism. The speed ratio of the transmission gear box ranges from $1 / 84$ to $1 / 14$, so that the rotating speed of the cranking mechanism is reduced to the range of $20 \mathrm{rpm}$ to $120 \mathrm{rpm}$. The reduced rotating speed has been measured by a photo-electric speedometer (Lineseiki TM-4013 \& TM-4000, accuracy $\pm 0.01 \%$ ). The variation of rotating speed is only $\pm 0.9 \%$. Therefore, the basic requirement of steady rotation is fulfilled.

\section{Cranking mechanism}

The cranking mechanism is constructed by renovating the above-mentioned single-cylinder horizontal engine. It comprises mainly a primary cylinder, a primary piston, a connecting rod, a camshaft, and a valve timing gear. Driven by the driving unit, the crankshaft rotates and the primary piston makes a reciprocating motion in the primary cylinder. The camshaft is connected to the crankshaft via a timing gear, which reduce the rotating speed of camshaft to one half of the crankshaft speed.

\section{Cylinder head}

The cylinder head is obtained from the abovementioned single-cylinder horizontal engine. Two lengthened push rods are installed, across the electromagnetic clutch and the test section, between the camshaft and the cylinder head. The opening and closing of intake and exhaust valves are maneuvered by the camshaft, with aids of the two push rods and the rocker arms on the cylinder head. The valve timing and the lift of valve stems are therefore retained as the same as the original engine. The original design of engine intake port is shaped to generate swirl motion during intake stroke. In order to study tumbling motion alone, the intake port has been modified so that swirl motion may be reduced to a minimum. Corresponding to the study interest, the intake valve may be shrouded to restrict the intake flow entering the cylinder in a particular direction.

Since the transparent cylinder contains incompressible water, there always exists a danger of cracking due to unexpected high in-cylinder pressure. A safety valve is therefore installed on the cylinder head to relieve excessive pressure and prevent the transparent cylinder from cracking. Air may be somehow entrained into the cylinder during the intake stroke. Some amount of bubbles in water is desirable for the seeding purpose. However, the size and the amount of bubbles need to be well controlled to ensure the simulation of the flow field inside an actual engine. A check valve is therefore installed on the cylinder head, such that large air bubbles will be driven out while outside air is prevented from entering.

\section{Electromagnetic clutch}

The primary piston drives the transparent cylinder. As the piston of the original engine, the primary piston undergoes all four strokes. Due to the incompressibility of the water, the compression and the expansion strokes must be skipped for the transparent piston. Therefore, during the compression and the expansion strokes, the connection between the primary piston and the transparent piston must be interrupted so that the transparent piston keeps stationary while the primary piston is moving. During the exhaust and the intake strokes, the connection between the primary piston and the transparent piston must be resumed to continue the cycle. These operations of intermediate disconnection and reconnection are attained by an electromagnetic clutch, a proximity sensor installed on the flywheel at the crank angle of BDC, and a programmable language controller. A maximum dragging force of $500 \mathrm{kgf}$ can be sustained for this electromagnetic clutch. Although the magnetic hysteresis cannot be avoided, it is within a tolerable range. When the primary piston moves to the BDC, about to undergo the compression stroke, the proximity sensor gives a signal to the controller to shut off the power supply of the electromagnetic clutch and therefore disconnect the transparent cylinder. Such that the transparent cylinder stays at the BDC while the primary cylinder still undergoes the compression and the expansion strokes. When the proximity sensor senses again that the primary piston moves to the BDC, about to undergo the exhaust stroke, the controller automatically turns on the power supply of the electromagnetic clutch to reconnect the transparent cylinder. Such that the transparent cylinder moves with the primary cylinder to undergo the exhaust and the intake strokes.

\section{Inlet and discharge system}

The inlet and discharge system consists of a water inlet tank and a discharge tank, both sized $0.3 \mathrm{~m}$ by $0.3 \mathrm{~m}$ by $0.6 \mathrm{~m}$. The intake port and the exhaust port of the cylinder head are connected, respectively, to the inlet tank and the discharge tank by flexible and transparent tubes. During the intake stroke, air bubbles may be entrained if the increasing speed of cylinder volume is faster than the inlet speed of water. Two-phase flow 
then occurs in the transparent cylinder that not only makes experimental observations difficult, but also distorts the in-cylinder flow field. The inlet tank is therefore placed at a level about $1 \mathrm{~m}$ higher than the test unit in an effort to increase the water inlet speed by the aid of gravity. Similarly, the discharge tank is placed at a level about $1 \mathrm{~m}$ lower than the test unit, to allow a faster discharge speed of water from the transparent cylinder to the discharge tank. The water in the discharge tank is pumped to the inlet tank at a higher level with a submerging pump. Thus, a circulating cycle of the working fluid is accomplished.

\section{EXPERIMENTS}

\section{Experimental Techniques}

Laser flow visualization is adopted to qualify the flow field and help the global understanding of the flow structure inside the cylinder. Particle image analyzer (PIA) is utilized to quantify the flow information. Laser flow visualization is in fact a part of the particle image analyzing technique and therefore will not be separately discussed. The particle image analyzer developed by Chen and Chou (1998) is modified in the present study.

The laser source adopted is a continuous mode laser (SP2017, 4W argon ion laser). The laser beam goes through an acousto optic modulator (AOM) before being transferred by mirror sets. A mirror is placed at the path of the first-order-diffracted beam, which only exits when the AOM is power supplied. The sequential laser pulsing can then be obtained by pulsating the power supply to AOM. Laser beam is then split into two equal-intensity beams by a splitter. These two laser beams will be directed to both sides of the test section through mirror sets. Two laser sheets of the same thickness are generated as the laser beams pass through two sheet generators located at the opposite sides of the test section, respectively. Laser sheeting from one side will usually cause the non-uniform illumination problem, which either produces a blurring area close to the wall or results in an insufficient light intensity in the far side. It is found that two sheets projected from each side of the cylinder helps significantly in resolving the illumination difficulty. The laser sheet generator is composed of a focus lens and two cylindrical lenses. Laser sheet of thickness from 1 to $10 \mathrm{~mm}$ can be produced by adjusting the distance between these two cylindrical lenses. In the current study, a laser sheet of $2 \mathrm{~mm}$ thickness is projected.

The wake flow field is visualized and recorded by a video camera. Both a Nikon F90x series camera and a high-resolution, $768 \times 494$ pixels CCD array with 30 $\mathrm{Hz}$ frame transfer rate are applied to perform the flow visualization task. The shutter speed and the $f \#$ will be adjusted based on the engine speed for the best resolution. A Dalsa CCD image sensor of a $226 \mathrm{~Hz}$ frame-transferrate is utilized to capture the images for the analysis of PIA system. The resolution $(256 \times 256$ pixels $)$ of the Dalsa CCD camera is found sufficient for the present experimental settings. Exposure time of the Dalsa CCD camera has been fixed at $1 / 226$ second, which will result in particle images being in the "streak" form. This streak form is suitable for the flow visualization but will cause difficulties in the analysis using PIA system. The above-mentioned AOM is applied for the accomplishment of light pulsing in an effort to obtain particle images of the fine "point" shape. This modulator (NEOS $\mathrm{N}-23080$ ) is operated at $80 \mathrm{MHz}$ frequency with a rise/ fall time of $100 \mathrm{~ns}$ and a diffraction efficiency of $80 \%$. A control unit is designed to function the power pulsing to the modulator. Output from the master clock of Dalsa image sensor is utilized to work as the synchronization reference. During each pulse time a square wave of 1volt amplitude is generated to initialize the driver system to power on the AOM. Pulse width in each frame is adjustable from $1 \mu$ s to $10 \mathrm{~ms}$ upon the velocity magnitudes to be measured and the illumination condition. A combination of 0.6 to $1 \mathrm{~W}$ laser power and 1 to $2 \mathrm{~ms}$ pulse width is applied under the present test conditions.

Many tiny air bubbles present inherently in the water-analogy engine. These tiny bubbles of $100 \mu \mathrm{m}$ diameter (measured by PIA) can serve as the seed particles. In order to describe the liquid motion based on the motion of tiny bubbles, the assumption of negligible slip velocity between each phase must be satisfied. According to Mei (1997), the particle response time can be expressed as

$$
t_{p}=\frac{2}{9} \frac{\left(\rho_{p} / \rho_{f}+1 / 2\right) a_{p}^{2}}{v_{f}}
$$

where $a_{p}$ is the diameter of bubble, $\rho_{p}$ is the density of seeding bubbles Since most of those tiny bubbles are of less than 100 microns, the calculated particle response time scale is at the order of $10^{-3} \mathrm{~s}$ which is much smaller than the characteristic time scale $\left(\approx 1 / \omega_{c} \approx 1 \mathrm{~s}\right)$ of the model engine. It suggests that the bubble should response quickly enough to the change of the flow. The amplitude of frequency response function can be calculated as (Mei, 1997)

$$
\left|H\left(\omega_{s}\right)\right|^{2}=\frac{(1+S)^{2}+\left(S+2 / 3 S^{2}\right)^{2}}{(1+S)^{2}+\left[S+2 / 3 S^{2}+4 / 9\left(\rho_{p} / \rho_{f}-1\right) S^{2}\right]^{2}}
$$

where $\omega_{s}$ is a fluctuation frequency and $S$ is the Stokes number which may be estimated as 


$$
S=\sqrt{\frac{\omega_{c} a_{p}^{2}}{2 v_{f}}},
$$

by assuming that the fluctuation is mainly resulted from the motion of the piston. It is found that $S$ is around 0.06 and the amplitude of frequency response function is very close to unity, which implies an excellent response of seeding bubbles to the flow. All of them support that the non-slip assumption is acceptable.

A $15 \times 15 \mathrm{~cm}^{2}$ field of view is chosen to cover the entire stroke without losing the detection of the microscopic flow structure. For each stroke, there are 240 and 135 frames recorded for operations at low (400 rpm) and high (1100 rpm) engine speeds, respectively. An Oculus $2048 \times 1024 \times 16$ bit frame grabber board is used to digitize the analog voltage. This frame grabber has a maximum storage ability of up to 256 frames on board. A PIA interrogation program is developed to identify particle images, locate centroids of particle images and compute displacement between image pairs. The instantaneous velocities and accelerations of seeding particles are determined from the calculation of particle displacement in frames. Note that the "instantaneous" velocities are calculated based on the movement of particles during three consecutive frame intervals, namely, 2/226 second. The calculated velocity vectors are then assumed as the instantaneous ones for the first frame. Under the current field of view, each seeding particle will occupy around one pixel and the uncertainty in locating the centroid can be reasonably estimated as $\pm 1 / 2$ pixel. Since most of the detected particle displacements are over 4 pixels, the estimated errors of velocity vectors are around $12 \%$. The instantaneous velocity field is displayed in a vector form originating from the particle location in the first frame.

The phase-averaged velocity field may correspond to the instantaneous one as long as the change of the flow structure in each cycle is not too significant. For each phase (i.e., at the same ATDC angle) under every stroke, there will be around 15 to 25 vectors detected after the PIA analysis. Therefore, the velocity field for each phase will contain at least 180 original PIA-detected velocity vectors by combining data acquired individually from 12 strokes. By doing so, it makes the interpolation process more realistic. A commercially available program, TECPLOT, is utilized for post-processing the PIA data. For performing the interpolation, the recorded flow field is divided into $30 \times 30$ grids, which results in a grid size of less than $3.3 \times 4.2 \mathrm{~mm}^{2}$ and an interpolation expansion ratio of 5 . The interpolation is then executed using the Kriging algorithm. The $z-$ component vorticities $\omega(i, j)$ of each grid point is calculated based on a finite difference approximation for the interpolated velocity field as

$$
\begin{aligned}
\omega(i, j) & \approx-\frac{\Delta u}{\Delta y}+\frac{\Delta v}{\Delta x}=-\frac{u(i, j+1)-u(i, j-1)}{2 \Delta y} \\
& +\frac{v(i+1, j)-v(i-1, j)}{2 \Delta x} .
\end{aligned}
$$

Here $u(i, j)$ and $v(i, j)$ are the respective velocity components in $x$ and $y$ directions at the interrogation grid point $(i, j) ; \Delta x$ and $\Delta y$ are the interrogation grid intervals in $x$ and $y$ direction, respectively. In this study, the $x-y$ plane represents the central axial plane. The strength of tumbling motion, represented by the circulation $\Gamma$, can be calculated from integrating those values of vorticity over the interrogation area. This is performed by using a finite integration formula

$$
\Gamma \approx \sum_{i=1}^{m} \sum_{j=1}^{m} \omega(i, j) \Delta x \Delta y,
$$

where $m$ and $n$ are the total grid numbers in the $x$ and $y$ directions, respectively.

\section{Experimental Conditions}

In the present study, the water analogy system are operated at two motor speeds, 20 and $55 \mathrm{rpm}$, equivalent to the engine speeds of 400 and $1100 \mathrm{rpm}$ in an actual engine, respectively. Based on the same clearance volume, piston with a cylindrical shape bowl is constructed. This bowl-in-piston is of $90 \mathrm{~mm}$ diameter, $46 \mathrm{~mm}$ bowl diameter and $23 \mathrm{~mm}$ bowl high. A piston without bowl is also tested for comparison. The effect of shrouded intake valve on the formation of tumbling motion is also under investigation. This shroud is extended to cover half-circular periphery of intake valve opening area. Tumbling motions for the inlet valve with the shrouded side placed toward either the cylinder wall or the center of cylinder are studied. The test conditions are summarized in Table 2.

\section{RESULTS AND DISCUSSION}

\section{Flow Visualization}

The laser sheets are projected along the central axial plane of cylinder and therefore the flow information recorded is the two-dimensional projection of the three-dimensional motion. Components of the circulation motion in this axial plane are considered to represent the tumbling motions. The internal flow field of engine is no doubt three-dimensional. To resolve the general three-dimensional flow structure needs an integration of the information extracted from many laser sheet planes projected at different locations. It is, however, not the focus of the current work. 
Table 2. Experimental conditions

\begin{tabular}{|c|c|c|c|c|c|c|c|}
\hline \multirow[b]{2}{*}{ experiments } & \multicolumn{2}{|c|}{ Piston } & \multicolumn{2}{|c|}{ Equivalent engine speed } & \multicolumn{3}{|c|}{ Shrouded intake valve } \\
\hline & with bowl & w/o bowl & $1100 \mathrm{rpm}$ & $400 \mathrm{rpm}$ & no & outer & inner \\
\hline I & $x$ & & $x$ & & & $x$ & \\
\hline II & $x$ & & & $x$ & & $x$ & \\
\hline III & $x$ & & $x$ & & & & $x$ \\
\hline IV & $x$ & & & $x$ & & & $x$ \\
\hline $\mathrm{V}$ & $x$ & & $x$ & & $x$ & & \\
\hline VI & $x$ & & & $x$ & $x$ & & \\
\hline VII & & $\times$ & $x$ & & & $x$ & \\
\hline VIII & & $\times$ & & $x$ & & $x$ & \\
\hline IX & & $x$ & $x$ & & & & $x$ \\
\hline$X$ & & $x$ & & $x$ & & & $x$ \\
\hline $\mathrm{XI}$ & & $\times$ & $x$ & & $\times$ & & \\
\hline XII & & $x$ & & $x$ & $x$ & & \\
\hline
\end{tabular}
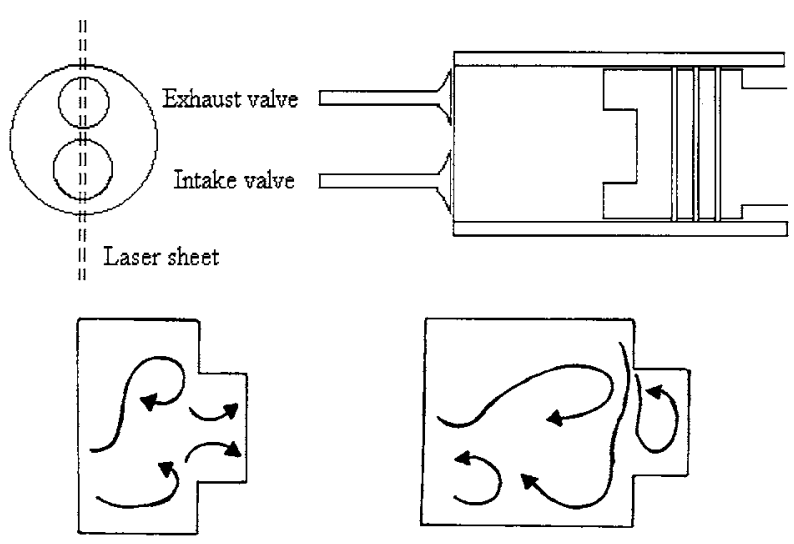

$\operatorname{ATDC} 70^{\circ}$

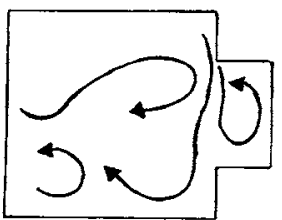

$\operatorname{ATDC} 140^{\circ}$

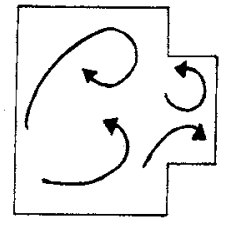

$\operatorname{ATDC} 110^{\circ}$

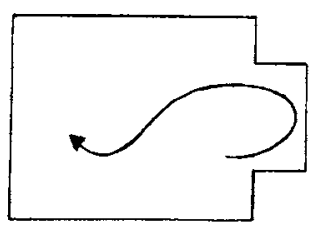

$\operatorname{ATDC} 180^{\circ}$
Fig. 2. Schematic in-cylinder flow field at different crank angle (equivalent engine speed = $1100 \mathrm{rpm}$, bowl-in-piston, non-shrouded intake valve).

\section{Non-shrouded intake valve}

The in-cylinder flow structure in the axial plane, generated by a non-shrouded intake valve, during the intake stroke is schematically shown in Figs. 2 and 3. Figure 2 shows the typical results for a bowl-in-piston while Fig. 3 demonstrates the flow structure for a flat piston. Fluid is entrained in a jet flow manner through the gap of non-shrouded inlet valve when it is open for

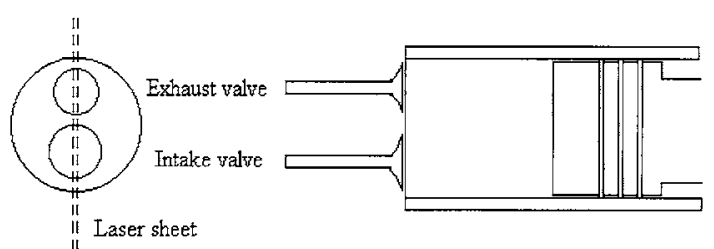

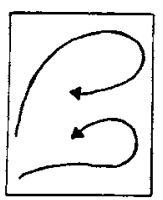

$\operatorname{ATDC} 110^{\circ}$

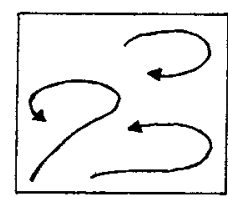

$\operatorname{ATDC} 150^{\circ}$

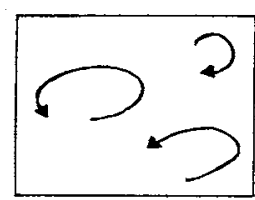

$\operatorname{ATDC} 180^{\circ}$
Fig. 3. Schematic in-cylinder flow field at different crank angle (equivalent engine speed $=1100 \mathrm{rpm}$, piston without bowl, non-shrouded intake valve).

the intake stroke. At low ATDC angles, the flow is dominated by two jet flows. These jet flows are deflected by the cylinder walls before ever reaching the piston. In the meantime, there are two small-size vortices of opposite rotating direction generated in the area close to the top of the bowl-in-piston. These vortices of small size will extend to fill up the bowl as demonstrated in the figure. Apparently, they are resulted from the phenomena of secondary flow. The large-scale vortices will follow the downward motion of piston to grow and well mix with each other. Since these two streams are apparently of the same strength, the well mixing will result in the disappearance of the obvious tumbling motion. At the BDC, the flow seems doing a three-dimensional helical motion (Fig. 2). Note that there is no small-scale vortices observed inside the bowl at the BDC. Similarly, no characteristic tumbling motion can be observed for the flow field generated by the 
movement of a piston without bowl (Fig. 3). It may be concluded that the configuration of constructing a bowl in piston does not help in both the generation and the maintenance of the tumbling motion.

As the engine is operated at a low speed, the phenomena of well mixing of two streams of opposite circulation are not obvious. Two deflected streams will develop and extend to occupy the whole cylinder space as the piston is moving downwards. At the BDC, a weak vortical flow with negative vorticity (rotating clockwise) will emerge inside the bowl, which is different from what observed (no vortex inside the bowl) for the engine operated at a high speed. This vortex is coming from the secondary flow generated near the piston surface. For the piston without bowl, the development of two streams of opposite vorticities seems different. As demonstrated in Fig. 3, especially for large ATDC angles, a pair of vortices with opposite vorticities occupy the bottom space of cylinder. The vortex with positive vorticity emerging from the intake valve is suppressed the development of and force it to exist in the upper part of cylinder only. No tumbling motion with an obvious single cell of vortex is found. In general, no matter the piston is with or without a bowl, the in-cylinder tumbling motion generated by a non-shrouded intake valve is insignificant.

\section{Intake valve with a shrouded outer-half-periphery}

If the intake valve is shrouded for the half periphery toward the cylinder wall as shown in Fig. 4, the entrained fluid is forced to jet-flow toward the cylinder center. A global in-cylinder tumbling motion of beautiful vortical flow pattern immediately emerges at low crank angles. This stream of negative vorticity will expand directly into the bowl and fill up the whole cylinder. The vortical flow will descend following the downward motion of the piston. A small-scale vortex of positive vorticity starts to appear in the corner near the exhaust valve when the ATDC angle reaches $100^{\circ}$. This vortex apparently is a product of the secondary flow and is observed to maintain the motion and the size throughout (until the piston reaching the BDC). The radial expansion of the tumbling motion with negative vorticity will be retarded due to the existence of this secondary flow in the corner. The axial expansion of the global tumbling motion into the bowl is also impeded due to the secondary flow generated near the top surface of the piston starting from the ATDC angle around $130^{\circ}$. This small-scale vortex of positive vorticity will occupy the whole bowl and retard the downward motion of the large-scale vortical flow. When the engine is operated at the low engine speed, generation of the secondary flow near the surface of piston is promoted for small

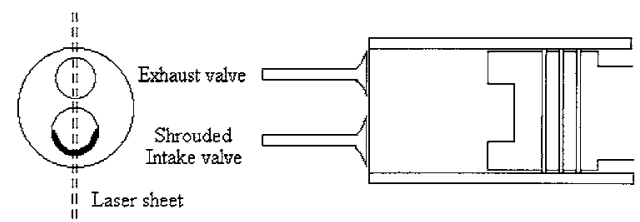

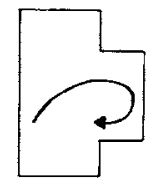

$\operatorname{ATDC} 45^{\circ}$

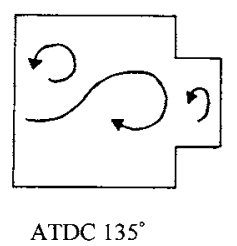

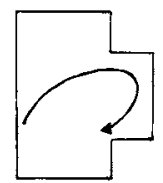

$\operatorname{ATDC} 66^{\circ}$

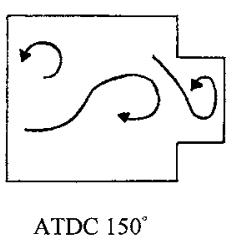

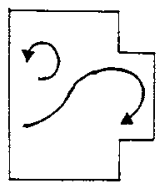

$\operatorname{ATDC} 100^{\circ}$

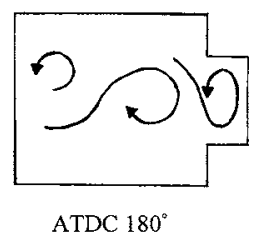

Fig. 4. Schematic in-cylinder flow field at different crank angle (equivalent engine speed = $1100 \mathrm{rpm}$, bowl-in-piston, intake valve with a shrouded outer-half-periphery).

crank angle. In the meantime, opposite to the situation at the high engine speed, no obvious small-scale vortex is observed in the corner near the exhaust valve. Therefore, the vortex of the tumbling motion can expand to occupy the whole space of cylinder. The expansion of this global vortical structure will render the existence of small vortices of positive vorticity only in the bowl space. Especially, at the BDC, the vorticity supplied by the secondary flow to the vortex in the bowl seems being entirely terminated. This observed incylinder flow structure is obviously different from the one when the engine is operated at the high speed.

Figure 5 demonstrates the in-cylinder flow structure generated for a piston without bowl schematically. Even operating at the high engine speed, there is no apparent small-scale vortex of positive vorticity in the corner near the exhaust valve. Instead, small-scale vortical streams due to the secondary flow appear nearing the top surface of piston. After the crank angle touches $150^{\circ}$, this secondary stream grows fast and retards the downward motion of the global reaching stream. At the BDC, these two vortices of opposite vorticities are apparently of the similar size and strength. As the engine is operated at the low speed, the intakegenerated vortical structure will have the time to follow the motion of piston and expand sufficiently in both radial and axial directions. The secondary flow near the surface of piston is also considerably suppressed. Not until the piston almost reaches the BDC will these small-scale vortices of positive vorticity be observed. 


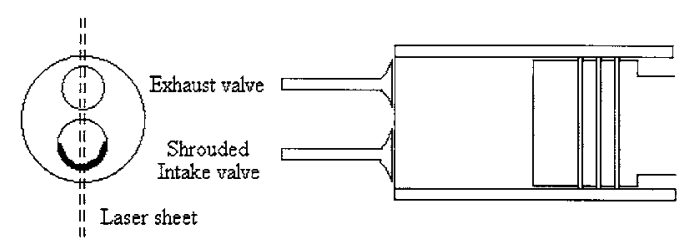

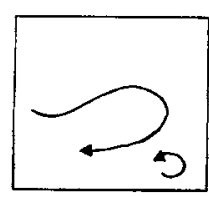

$\operatorname{ATDC} 150^{\circ}$

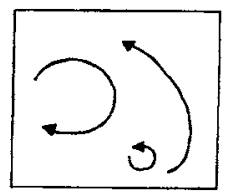

$\operatorname{ATDC} 160^{\circ}$

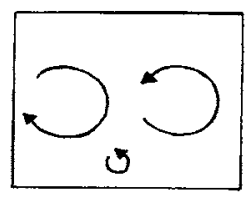

$\operatorname{ATDC} 180^{\circ}$
Fig. 5. Schematic in-cylinder flow field at different crank angle (equivalent engine speed $=1100 \mathrm{rpm}$, piston without bowl, intake valve with a shrouded outer-half-periphery).

A clear in-cylinder tumbling motion with a beautiful vortical structure can be easily identified when the piston is at the BDC.

The visualization result suggests that the addition of shroud to the intake valve will help the generation of large-scale in-cylinder tumbling motion. A small-scale vortical motion due to the secondary flow will be plunged into the bowl in piston. The growth of this secondary flow is therefore retarded. In this way, especially for operation at the high engine speed, a vortical structure in the axial plane can be maintained. However, it requires further verification whether the small-scale tumbling structure in the bowl really benefits the generation of turbulence during the compression stroke.

\section{Intake valve with a shrouded inner-half-periphery}

Since the intake valve is shrouded for the half periphery toward the cylinder center as shown in Fig. 6, the entrained flow is deflected to enter the cylinder along the cylinder wall. This makes the flow to bear more viscous-dissipation due to the wall effect. However, this effect does not guarantee the generation of vortex of a single vorticity only. In fact, under the high engine speed, the fluid will immediately split into two major streams. These two streams are of opposite vorticities (Fig. 6). The stream of positive vorticity will expand radially toward the other half part of cylinder. In the meantime, in the corner near the exhaust valve starts to see streams of negative vorticity generated due to the secondary flow phenomena. The stream of negative vorticity coming from the intake valve will essentially expand axially to quickly reach the bowl in piston. The vortex will grow and move, as the piston is moving downwards. Due to the existence of this vortical stream

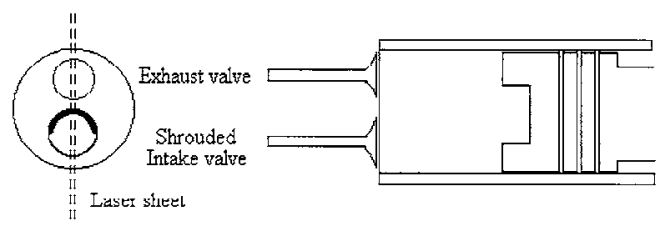

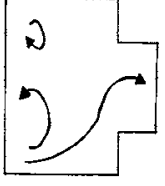

$\operatorname{ATDC} 100^{\circ}$

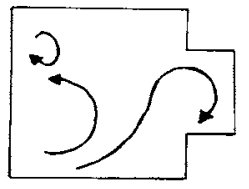

$\operatorname{ATDC} 140^{\circ}$

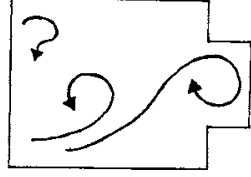

ATDC $180^{\circ}$
Fig. 6. Schematic in-cylinder flow field at different crank angle (equivalent engine speed $=1100 \mathrm{rpm}$, bowl-in-piston, intake valve with a shrouded inner-half-periphery).

of negative vorticity, the axial expansion of another stream of positive vorticity is therefore retarded and forced to stay in the upper part of the cylinder. The observation of small-scale vortex in the corner near the exhaust valve is not so apparent as the engine is operated at the low engine speed. Since the piston is moving at a relatively low speed, the introduced vortical stream of positive vortex will have enough time to expand. Therefore, it will gradually occupy the whole cylinder space as the piston is pulling downwards. In the meantime, a stream of negative vorticity and much weaker strength is generated in the vicinity of the top surface of piston. The expansion of the global vortical flow will restrict the movement of this small-scale vortex and eventually force its motion within the territory of bowl only.

The split of entrained streams of opposite vorticities no longer exists in the in-cylinder flow field generated with a piston without a bowl. The vortical flow of positive vorticity expands steadily in both the radial and the axial directions. No small-scale vortices generated by the secondary flow are observed for the ATDC angle up to around $50^{\circ}$. After this ATDC angle, small-size vortical flow of negative vorticity appears steadily near the top surface of piston. No permanent small-scale vortices are found in the region near the exhaust valve. Note that it is opposite to that found for the flow field generated by a bowl-in-piston. As the engine is operated at the low speed, a tumbling motion with a solid vortical flow pattern can be seen through all the crank angles for the flow field generated by a piston without bowl. This global vortex is generated immediately after the flow leaves the intake valve. It grows and moves downwards following the movement of piston. Smallscale vortices may be occasionally found near the exhaust valve or the top surface of piston. However, it 

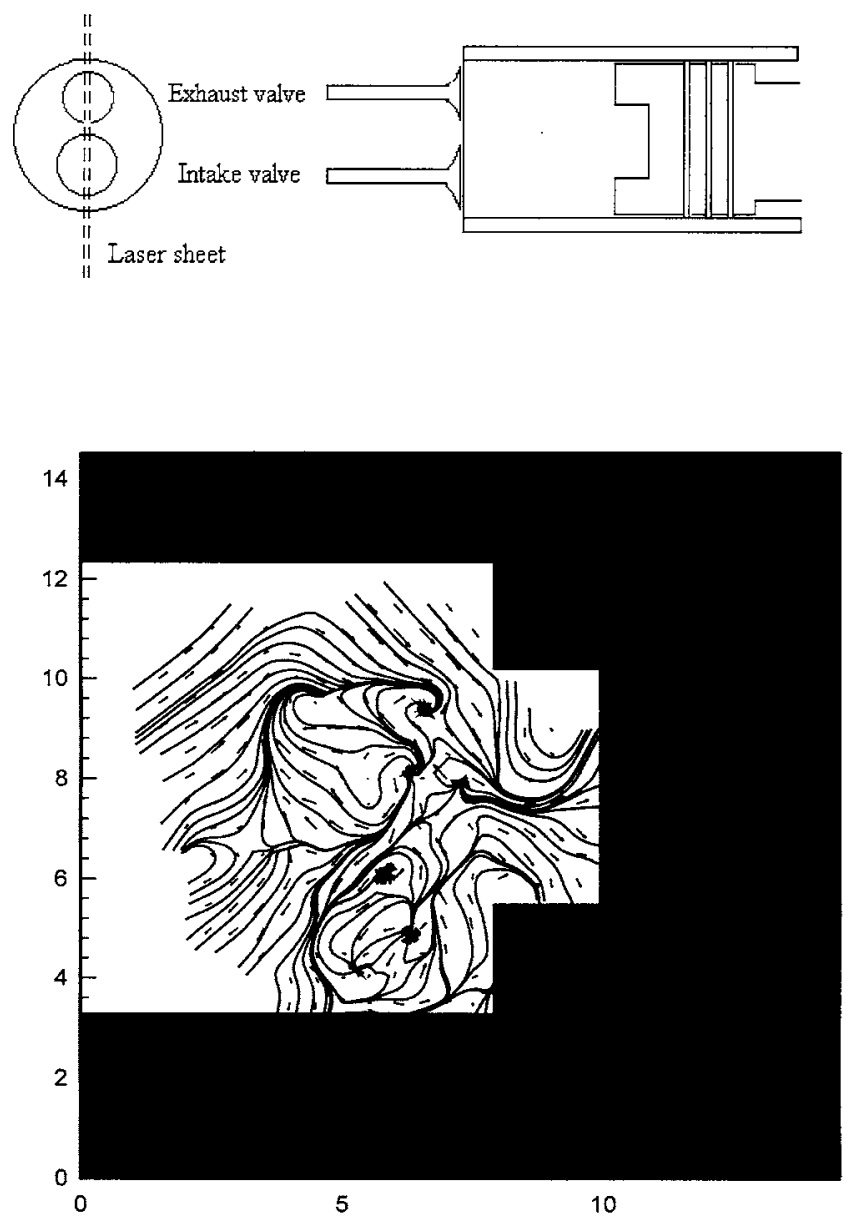

Fig. 7. Interpolated velocity field and integrated streamline pattern of the in-cylinder flow field (equivalent engine speed $=1100 \mathrm{rpm}$, bowlin-piston, non-shrouded intake valve, $120^{\circ}$ ATDC).

diminishes quickly.

Again, the addition of shroud to the intake valve will help the generation of large-scale in-cylinder tumbling motion. A small-scale vortical flow will be reserved inside the bowl in piston. The use of bowl-inpiston shows no immediate help in the generation and the maintenance of the vortical flow pattern in the axial plane. In fact, it appears that the tumbling motion in the in-cylinder flow field generated by the piston without bowl is even more prominent.

\section{PIA Sample Results}

The interpolated PIA-detected in-cylinder velocity fields with integrated streamlines and the vorticity contours derived from the above interpolated flow field are demonstrated in Figs. 7 and 8, respectively. This incylinder flow field is generated with a non-shrouded intake valve under the operation at the high engine speed (1100 rpm, equivalently). The crank angle is
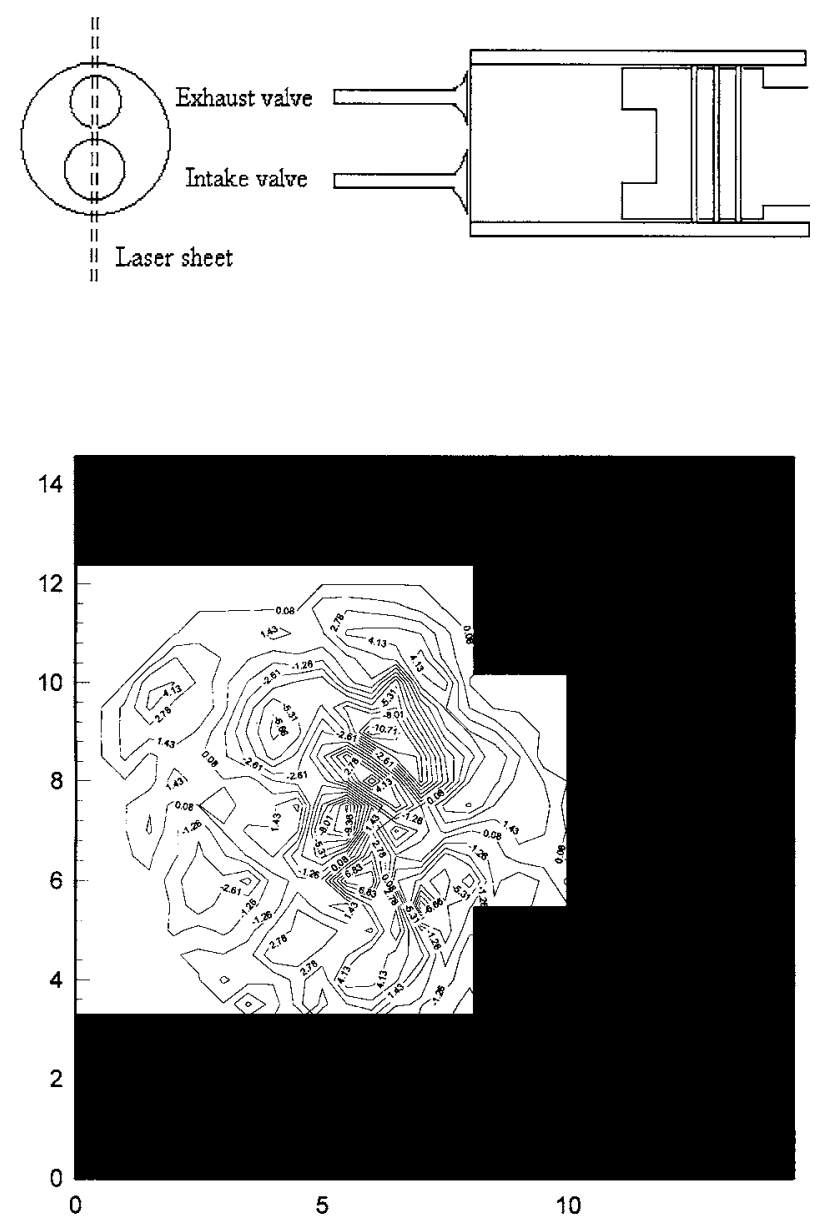

Fig. 8. Vorticity contours of the in-cylinder flow field (equivalent engine speed $=1100 \mathrm{rpm}$, bowl-in-piston, non-shrouded intake valve, $120^{\circ}$ ATDC).

$120^{\circ}$ ATDC. Since a PIA detected vector is extracted from the displacement of a seeding particle in three consecutive frames, the time-period involved will be $2 /$ $226 \mathrm{sec}$. The crank angle involves in this short period is around $3^{\circ}$. The reflection of laser sheet is so strong in the region near the cylinder wall that a serious imageblurring problem is inherently resulted. Also, the jet flow in the region near the intake valve is highly threedimensional. Therefore, the seeding particles will not maintain their traces on the laser sheet during the period $(2 / 226 \mathrm{sec}$.) for capturing three images. Additionally, the concentration of entrained bubbles in this region is highly intensive, which renders the capture of image impossible. The formation and dissipation mechanism of the tumbling motion can be understood through thorough topological study for the PIA-detected flow fields. In Fig. 7 one finds many topological characteristics such as the stable and the unstable foci, the saddle point, the bifurcation lines and etc. However, most of them only exist for the three-dimensional flow field, which 
strongly implies that the in-cylinder flow structure is highly three-dimensional. The flow structure extracted from the PIA measurements in this figure show resemblance to the flow visualization result shown in Fig. 2, which support the proper set-up of PIA in the engine research. However, analysis of the quantitative flow information through the PIA results is beyond the scope of this paper. From the contours of vorticity, it can be known that the flow is not fully turbulent. The vorticity is concentrated on the location where the foci and the saddle points being located. The vorticity decays fast as one moves away from the foci or the saddle points. It also clearly shows that there is a weak vortical flow near the top surface of piston, and that this flow expands deeply into the bowl. The secondary flow phenomena are obviously demonstrated. Integrating the vorticity of Fig. 8 using Eq. (14), one finds that the magnitude of the circulation is nearly zero. It implies that the flow through the non-shrouded intake valve is irrotational. No obvious tumbling motion is available for such configuration. It demonstrates that one can use the vortex strength (i.e., circulation) to identify the quality of the tumbling motion. Nevertheless, a tumbling motion of "good" quality should depend on the contribution of generating turbulence during the compression stroke. This point remains further investigation.

\section{CONCLUDING REMARKS}

The potential of using a video-based particle image system in studying the in-cylinder tumbling flow structure of an engine is demonstrated. No significant tumbling motion is found accompanying with the use of a non-shrouded intake valve. Adding a shroud to the intake valve will help the generation of large-scale vortical tumbling motion. A small-scale vortex will be reserved inside the bowl in piston. The use of bowl-inpiston may or may not help in the generation and the maintenance of the tumbling flow pattern depending on the part of intake valve shrouded. The study of the topological characteristics of the PIA-detected flow fields can help significantly in the understanding of the formation and dissipation mechanism of the tumbling motion. Further investigation to relate the quality of the vortical flow in the axial plane with the generation of turbulence during the compression stroke is strongly recommended.

\section{ACKNOWLEDGEMENTS}

This work was supported by the National Science Council, R.O.C. under Grant \#86-2212-E-019-005 and \#87-2212-E-019-008.

\section{NOMENCLATURE}

$a_{p} \quad$ the diameter of seeding bubble

$D$ bore diameter

Eu Euler number

$f \#$ inverse of the relative aperture of video camera (= focal length/aperture)

$H$ frequency response function

$M \quad$ Mach number

$m \quad$ total grid numbers in the $x$ direction

$n \quad$ total grid numbers in the $y$ direction

$p \quad$ pressure

Re Reynolds number

$U_{p} \quad$ average piston speed

$u \quad$ velocity component in the $x$ direction

$V \quad$ velocity

$v \quad$ velocity component in the $y$ direction

$S \quad$ Stokes number

St Strouhal number

$t$ time scale

$t_{p} \quad$ particle response time

$x \quad$ horizontal upward distance from the bottom-left corner of image frames

$y \quad$ vertical right-hand distance from the bottom-left corner of image frames

$\Delta x \quad$ interrogation grid interval in the $x$ direction

$\Delta y \quad$ interrogation grid interval in the y direction

$\Gamma \quad$ circulation

$v_{f} \quad$ kinematic viscosity of liquid

$\omega \quad$ vorticity component in the $z$ direction

$\omega_{c}$ angular velocity of the crankshaft

$\omega_{s} \quad$ frequency of fluctuation

$\rho_{f} \quad$ density of liquid

$\rho_{p} \quad$ density of seeding bubble

\section{REFERENCES}

1. Arcoumanis, C., Hu, Z., Vafidis, C., and Whitelaw, J.H., "Tumbling Motion : A Mechanism for Turbuience Enhancement in Spark-Ignition Engines," SAE paper 900060, (1990).

2. Belair, R.C., Davis, R.G., Kent, J.C., and Tabaczynski, R.J., "Combustion Chamber Effects on Burn Rates in a High Swirl Spark Ignition Engine," SAE paper 830335 , (1983).

3. Chen, R.C., and Chou, I.S., "Preliminary Studies of Bubble Dynamics Using Particle Image Analyzer," $J$. CSME, Vol. 19, No. 5 (1998).

4. Duggal, V.K., Alternative Fuel Engine Programs, Cummins Engine Company, Inc. (1996).

5. Evans, R.L., and Blaszczyk, J., "A Comparative Study of the Performance and Exhaust Emissions of a Spark Ignition Engine Fueled by Natural Gas and Gasoline," Proc., Instn. of Mech. Engrs., Vol. 211, part D, Feb. 
(1997).

6. Heywood, J.B., "Fluid Motion within the Cylinder of Internal Combustion Engines-The 1986 Freeman Scholar Lecture," J. of Fluids Eng., Vol. 109, pp. 3-35 (1987).

7. Heywood, J.B., Internal Combustion Engine Fundamentals, McGraw-Hill, New York, (1988).

8. Kent, J.C., Mikuiec, A., Rimai, L., Adamczyk, A.A., Mueller, S.R., Stein, R.A., and Warren, C.C., "Observations on the Effects of Intake-Generated Swirl and Tumble on Combustion Duration," SAE paper 892096 , (1989).

9. Khalighi, B. and Huebler, M.S., "A Transient Water Analog of a Dual-Intake-Valve Engine for Intake Flow Visualization and Full-Field Velocity Measurements," SAE paper $880519,(1988)$.

10. Khalighi, B., "Intake-Generated Swirl and Tumble Motions in a 4-Valve Engine with Various Intake Configurations-Flow Visualization and Particle Tracking Velocimetry," SAE paper 900050, (1990).

11. Khalighi, B., "Study of the Intake Tumble Motion by Flow Visualization and Particle Tracking Velocimetry," Experiments in Fluids, Vol. 10, pp. 230-236 (1991).

12. Khalighi, B., "Multidimensional In-Cylinder Flow Calculations and Flow Visualization in a Motored Engine," J. of Fluids Eng., Vol. 117, pp. 282-288 (1995).

13. Ma, T., Davis, M., and Collings, N., "Low Speed Dynamic Similarity Modeling in Internal Combustion Engines," SAE paper 860239, (1986).

14. Mei, R., "Velocity Fidelity of Flow Tracer Particles," Experiments in Fluids, Vol. 22, pp. 1-13 (1997).
15. Willas, D.A., Meyer, W.E., and Birnie, C., "Mapping of Airflow Patterns in Engines with Induction Swirl," SAE paper 660043, (1966).

16. Zucrow, M.J., and Hoffman, J.D., Gas Dynamics, John Wiley, New York, (1976).

\section{杯狀活塞引擎進氧産生滾轉運動之 探討}

鄭元良陳第哲張照和

國立臺灣海洋大學機械與輪機工程研究所

摘 要

最近倡議利用滾轉運動增強引擎中之紊流強 度, 以增進燃燒效率與降低運轉變異性。本研究之目 標即在於研究杯狀活塞柴油機中之滾轉流場結構。爲 克服引擎内流場因流速太快而不易實施流場觀測之困 難, 本研究先依據類比分析的結果, 設計並組裝完成 一部能模擬杯狀活塞柴油機, 但運轉速率較慢之透明 水缸類比模擬系統。藉由此透明水缸類比模擬系統之 運轉, 配合快速攝影系統的使用, 成功地達成初步之 定性流場觀測結果, 進而瞭解滾轉流場之時變性。同 時應用高速質點影像测速儀, 成功地定量量測瞬時之 流轉流場, 證明本引擎類比系統及量測設施之適當性 與未來之研究潛能。 